\title{
Determining the influence of Gaussian blurring on inversion effects with talking faces
}

\author{
SHARON M. THOMAS and TIMOTHY R. JORDAN \\ University of Nottingham, Nottingham, England
}

\begin{abstract}
Perception of visual speech and the influence of visual speech on auditory speech perception is affected by the orientation of a talker's face, but the nature of the visual information underlying this effect has yet to be established. Here, we examine the contributions of visually coarse (configural) and fine (featural) facial movement information to inversion effects in the perception of visual and audiovisual speech. We describe two experiments in which we disrupted perception of fine facial detail by decreasing spatial frequency (blurring) and disrupted perception of coarse configural information by facial inversion. For normal, unblurred talking faces, facial inversion had no influence on visual speech identification or on the effects of congruent or incongruent visual speech movements on perception of auditory speech. However, for blurred faces, facial inversion reduced identification of unimodal visual speech and effects of visual speech on perception of congruent and incongruent auditory speech. These effects were more pronounced for words whose appearance may be defined by fine featural detail. Implications for the nature of inversion effects in visual and audiovisual speech are discussed.
\end{abstract}

The facial movement that accompanies speech production is a particularly powerful component of human communication because of its influence on speech perception. Seeing the face of a talker can improve intelligibility in a noisy environment (Erber, 1969; MacLeod \& Summerfield, 1987, 1990; Middleweerd \& Plomp, 1987; Sumby \& Pollack, 1954) and help recover a difficult message even when the auditory signal is clear (Reisberg, McLean, \& Goldfield, 1987). Furthermore, in the McGurk effect (e.g., McGurk \& MacDonald, 1976), different auditory and visual inputs combine to form a new percept that was not presented in either modality alone, or visual input dominates auditory input. For example, when an auditory syllable (e.g., /ba/) is presented in synchrony with a visual image of a talker pronouncing a different syllable (e.g., /ga/), observers generally perceive a syllable (e.g., /da/) that was not presented either visually or auditorily but that represents a combination of both sources of information.

Previous research indicates that the processes responsible for the perception of facial movement during visual and audiovisual speech perception are considerably resistant to loss of visible information from the talker's face. For example, Jordan, McCotter, and Thomas (2000) reported that removing color from talking facial images does not affect visual speech identification or visual influences on identifying the auditory components of congruent and incongruent audiovisual speech. Audiovisual speech perception

We are grateful to Vicki Bruce and two anonymous reviewers for their comments concerning this article. Correspondence should be addressed to S. M. Thomas, School of Psychology, University of Nottingham, University Park, Nottingham, NG7 2RD, England (e-mail: smt@psychology. nottingham.ac.uk). performance also remains substantially unchanged across viewing angles, from full face to three-quarter to profile (Jordan \& Thomas, 2001; but see Kanzaki, Kato, \& Tohkura, 1998), and in the picture plane across orientations throughout 360-degree rotations (Jordan \& Bevan, 1997). Audiovisual speech perception has also proven to be relatively immune to the effects of increasing the distance at which faces are observed, and to decreases in facial size (Jordan \& Sergeant, 1998, 2000). In particular, the considerable resistance to reductions in image size suggests that the visual cues underlying visual speech perception with full-size faces are relatively coarse, so that even when viewed in a facial image just $10 \%$ of full size (Jordan \& Sergeant, 1998, 2000), the effectiveness of these cues remains unimpaired. The resilience of the visual and audiovisual speech processing system to loss of perceptual detail is more dramatically evidenced by studies using pointlight images, in which only small points of light are visible and in which no detailed pictorial information is present. The point-light technique has been used in a number of studies in which observers recognized the biological motion produced by moving point-light displays as a person performing specific activities (e.g., walking, running, jumping, lifting weights; Bingham, 1987; Johansson, 1973; Kozwolski \& Cutting, 1977) and has also been applied to faces; expression, age, and gender have been identified (Bassili, 1978; Berry, 1990). When used for talking faces (Johnson, Rosenblum, \& Saldaña, 1994; Rosenblum, Johnson, \& Saldaña, 1996; Rosenblum \& Saldaña, 1996), a static configuration of reflective dots placed on a blacked-out face could not be identified as a face, suggesting that not enough pictorial facial information was available for static face processing. Nevertheless, when moving, these pointlight displays were recognized as unimodal visual speech 
and exerted powerful influences on congruent (presented in noise) and incongruent auditory speech (Johnson et al., 1994; Rosenblum et al., 1996; Rosenblum \& Saldaña, 1996). However, across all these studies, point-light faces did not show the same degree of visual influence as fully illuminated face stimuli, so although the visual and audiovisual speech processing system can tolerate loss of visual information specifying facial features, these features do normally convey some useful information.

One aspect of the facial image that remains when perception of featural detail is impaired is facial configuration. The term configuration has been variously interpreted in recent years but generally refers to the interrelationship between individual facial features (for a full discussion of the varied and various interpretations of this term, see Searcy \& Bartlett, 1996; see also Leder and Bruce, 2000). In the present paper we will use the term configuration in its classic sense to refer to the spatial relationships between individual elements within the facial frame, such as the distance between jaw and nose, in contrast to the "piecemeal" consideration of each individual feature. Moreover, because we are considering the nature of visual speech, we are necessarily discussing moving faces, in which facial configuration can be dynamic-for example, changing positions of upper and lower lips.

In the context of static face recognition, the prevalent opinion is that face recognition is based on configural and featural modes of representation, but configural information may play a more important role (Carey \& Diamond, 1977; Rhodes, 1988; Sergent, 1984; Tanaka \& Farah, 1993). This notion is supported by the fact that facial information is more difficult to encode when this information is viewed in inverted (upside-down) faces (see Valentine, 1988, for a review). Effects of facial orientation on perception of visual and audiovisual speech have been investigated previously with variable findings. Jordan and Bevan (1997), using natural stimuli, showed that face orientation had no effect on the accuracy of reporting visual and congruent audiovisualsyllables. However, the accuracy of incongruent audiovisual speech report was affected by face orientation when the speech sounds had a different appearance when upright as opposed to inverted (e.g., visual /ba/, where vertical lower lip/chin movement is downward in upright faces and upward in inverted faces) but not affected when the speech sounds had a similar appearance when upright as opposed to inverted (e.g., visual /ga/, where movement of the lip corners is horizontal). Rosenblum, Yakel, and Green (2000) reported similar results for the incongruent auditory/va/ and the visual /ba/ stimuli, in their Experiments 1 and 3; in their Experiment 2, they found similar results for the auditory $/ \mathrm{ba} /$ and the visual /ga/, but not for the auditory /ba/, the visual/va/, or indeed the auditory $/ \mathrm{ga} /$ or visual $/ \mathrm{ba} /$. These last results are at odds with the notion that inversion effects obtain with articulations that look very different upright as opposed to inverted, since the stimulus / va/ involves distinct asymmetrical labiodental movements, and the stimulus /ga/ involves quite symmetrical velar movements. Jordan and Bevan suggested that only when the articulation of visual speech involves substantial facial movement does facial inversion reduce the effects of visual speech on auditory speech perception. They further suggested that this result implies an important role for configural information present in upright faces when substantial facial movements are involved in speech production. It is possible, however, that some dynamic spatial relationships among the lips, teeth, tongue, and a very restricted area around the mouth are encoded for audiovisual speech perception (Jordan \& Bevan, 1997). Inversion of the face in Jordan and Bevan's experiments may have disrupted the encoding of this finer facial movement information. This notion is supported by the finding of Rosenblum et al. (2000), who found inversion effects with a mouth-only stimulus articulating the syllable /ba/. With the mouth-only display, the relative motion information would be less observable than in a full face, where, for example, chin movement provides a large cue to direction of motion.

If it is the case that coarsely defined facial movement rather than the finer facial motion of lips, teeth, and tongue forms the basis of inversion effects, then one would expect that impeding perception of finer facial motion, while preserving perception of coarse facial motion, would result in the same pattern of inversion effects as that observed in Jordan and Bevan's experiments. The roles of these two different types of information (fine, coarse) in inversion effects may be usefully examined by manipulation of spatial frequencies. Previous research has suggested that when the spatial frequency information in an image is manipulated, coarse global features (e.g., configural relations) are processed by low spatial-frequency channels whereas fine, local features are processed by high spatial-frequency channels (De Valois \& De Valois, 1988). In the context of static face recognition, research suggests that people are sensitive to configural relations among features transmitted through low spatial-frequency channels. Natural faces without high spatial-frequency information are still recognizable (e.g., Bachmann, 1991; Costen, Parker, \& Craw, 1996) and facial affect perception is also highly robust to image degradation (Wallbott, 1992). Eliminating high spatial-frequency information will be informative with regard to whether perception of fine detail or coarse configural relations form the basis of inversion effects in visual and audiovisual speech perception. Some evidence does exist to suggest that spatial resolution of upright faces may be substantially reduced before affecting visual speech perception (Brookes \& Templeton, 1990; Campbell \& Massaro, 1997; Frowein, Smoorenburg, Pyters, \& Schinkel, 1991; Pearson, 1981; Vitkovitch \& Barber, 1996). Using spatial quantized displays of a synthetic talking head, Campbell and Massaro (1997) reported steady declines in visual speech reading performance with increasing spatial quantization, but performance was largely resistant down to and including 16 cycles per face width (f/w) displays. It remains to be seen how such reductions in spatial resolution affect visual and audiovisual speech perception with inverted faces. We examined this issue in the present 
experiments, where the chosen method of distortion was Gaussian blurring rather than spatial quantization, since spatial quantization introduces high spatial-frequency information that is not of the original image, and we wished to examine the importance of coarse, global facial information. Since no previous studies have measured the effects of blur on audiovisual speech perception, even with upright faces, we conducted a pilot study to assess the effects of increasing levels of facial blur on the strength of the McGurk effect.

In the pilot study, seven filtered versions of a talking face were generated by applying the original with a Gaussian filter having radii of $9,18,24,36,48,72$, and 144 pixels. These seven levels were equivalent to the following cycles per f/w; Level 1, 32 cycles per f/w; Level 2, 16 cycles per f/w; Level 3, 12 cycles per f/w; Level 4, 8 cycles per f/w, Level 5, 6 cycles per f/w, Level 6, 4 cycles per f/w, and Level 7, 2 cycles per f/w.

Nine unfiltered versions (288 cycles per $\mathrm{f} / \mathrm{w}$ ) of the incongruent /audio//visual/ pairings (bat//vat/, /din//dip/, /map//nap/) were shown intermingled with three examples of each of the seven filtered versions of each stimulus. These stimuli were chosen because they represent a range of facial movements during their articulations and involve variable amounts of facial detail for their perception. Articulation of /map/, for example, involves a bilabial separation followed by a downward jaw displacement, followed by a rapid bilabial closure. The teeth and tongue are not obvious, and the movement is substantial, so detailed information may not be important. Articulation of /din/, by contrast, involves relatively little movement, bringing the teeth together at the start of the word, accompanied by a horizontal movement of the corners of the mouth, fol- lowed by the tongue touching the top of the mouth. Articulation of /vat/ combines substantial movement with the visual detail of the teeth over the lower lip at the beginning of the word. Nine clips were also included in which no facial movements occurred (auditory signal only).

Mean correct responses across 5 participants are shown in Figure 1. An analysis of variance (ANOVA) with the factors of stimulus type (incongruent audiovisual pairings /bat//vat/, /din//dip/,/map//nap/) and cycles per f/w (288, $32,16,12,8,6,4,2)$ revealed a main effect of cycles per $\mathrm{f} / \mathrm{w}[F(7,28)=47.98, p<.0001]$. Post hoc tests (NewmanKeuls) produced the following comparisons between performance accuracy as cycles per $\mathrm{f} / \mathrm{w}$ decreased; $288=$ $32=16=12=8<6(p<.05)<4(p<.001)=2$. Comparisons with the auditory-only condition revealed that perception of the auditory signal/bat/ was impaired when the participant was presented with the incongruent visual speech stimulus /vat/ down to 8 cycles per f/w $(p \mathrm{~s}<.01)$, perception of the auditory stimulus /din/ was impaired when the participant was presented with the incongruent visual-stimulus/dip/ down to 6 cycles per f/w $(p \mathrm{~s}<.01)$, and perception of the auditory stimulus /map/ was impaired when the participant was presented with the incongruent visual stimulus /nap/ down to 12 cycles per $\mathrm{f} / \mathrm{w}$ $(p \mathrm{~s}<.01$; Newman-Keuls, $p$ s $<.01)$.

In summary, the pilot study demonstrated that even when perception of visual detail is severely reduced by Gaussian blurring, visual speech can exert a powerful influence on auditory speech. This result complements previous findings suggesting the efficacy of coarse visual cues in visual and audiovisual speech perception (e.g., Campbell \& Massaro, 1997). However, the decline in the effectiveness of visual speech below 8 cycles per $\mathrm{f} / \mathrm{w}$ in the present re-

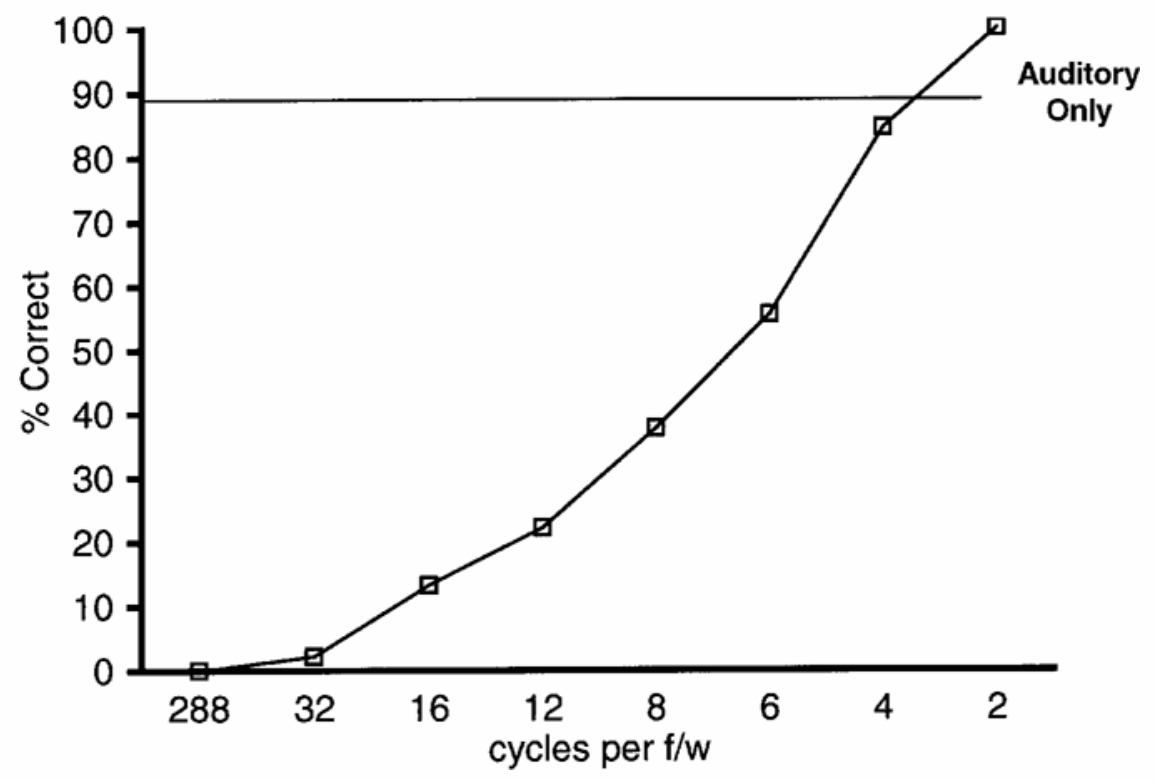

Figure 1. Mean percentage of auditory stimuli correctly identified in the pilot study for incongruent audiovisual displays at each blurring level. 
sults also indicates that higher spatial frequencies do carry some information in audiovisual speech recognition from upright faces, and the stimulus differences suggest further that the contributions of high and low spatial frequencies may be dependent on the individual nature of particular visible articulations. The extent to which these different spatial frequencies contribute to visual and audiovisual speech perception in inverted faces has yet to be resolved. By presenting blurred upright and inverted talking faces where perception of coarse, configural information is preserved, while perception of finer facial information is impeded, we may determine the contributions of coarse and fine information to inversion effects in visual and audiovisual speech perception. If it is the case that substantial (coarsely defined) facial motion rather than finer facial motion (e.g., of lips, teeth, and tongue) form the basis of inversion effects, then we would expect that impeding perception of finer facial motion, while preserving perception of coarse facial motion, would result in the same pattern of inversion effects previously observed with talking faces (e.g., Jordan \& Bevan, 1997). If, however, perception of finer facial information is important for the manifestation of inversion effects, then we may expect a different pattern of results when talking face displays are blurred.

\section{EXPERIMENT 1}

In Experiment 1 we examined the influence of different facial displays (unblurred, blurred, inverted, upright) in order to examine the role of visually coarse and fine information in inversion effects for visual and audiovisual speech recognition. Effects of orientation and blurring on the perception of visual speech per se and effects on auditory speech perception were examined using congruent audiovisual stimuli, in which visual speech matched the auditory signal (as in normal spoken interactions), and using incongruent (McGurk) audiovisual stimuli, in which visual speech was dubbed onto an incongruent auditory signal. McGurk effects were included because they offer a particularly stringent test of automatic and perceptual processing of audiovisual speech stimuli (e.g., Jordan \& Bevan, 1997; Jordan \& Sergeant, 1998, 2000; Liberman \& Mattingly, 1985; Rosenblum \& Saldaña, 1992; see also Rosenblum \& Saldaña, 1996).

\section{Method}

Participants. Ten native speakers of British English participated in a 1-h session. All participants had normal or corrected-to-normal vision and good hearing.

Stimuli. The stimulus items comprised articulations of six consonant-vowel-consonant words (/bat/, /din/, /dip/, /map/, /nap/, and /vat/). Stimulus sequences were created by videotaping the face of a 23-year-old female native English speaker onto digital videotape while she fixated the video camera. The speaker's face was fully illuminated and recorded against a dark background with only the face and neck visible. Recordings were made of the talker saying each of six words a number of times in a British English accent. Recordings were also made of the face making no face movements (with mouth closed). Each word was articulated in a natural way with no artifi- cial emphasis on face movements, and no extraneous movement (e.g., eye blinks) occurred beyond the articulation itself. From these recordings, one example of each of the six audiovisual words was selected, together with one example of the face with no facial movements (for the auditory-only condition), on the basis that all examples were matched for duration, intensity, and clarity.

The face of the talker measured 288 pixels horizontally and 340 vertically. Three filtered versions of each clip were generated by applying the original with a Gaussian filter having radii of 18,36 , and 72 pixels (Figure 2). Spatial frequency was varied at four steps from Level 0 (normal display). These four levels were equivalent to the following cycles per face: Level 0, 288 cycles per f/w; Level 1, 16 cycles per f/w; Level 2, 8 cycles per f/w; Level 3, 4 cycles per f/w. These levels of blur were chosen on the basis of results from the pilot study indicating changes in performance at these levels. Inverted versions of all the unfiltered and filtered clips were then created using the rotation function of the postproduction video-editing software (Radius Edit DV).

Each voice-face combination was presented in eight different displays: Upright Unblurred, Upright Blur Level 1, Upright Blur Level 2, Upright Blur Level 3, Inverted Unblurred, Inverted Blur Level 1, Inverted Blur Level 2, and Inverted Blur Level 3. The auditory, visual, and audiovisual conditions were achieved by editing the selected examples of the audiovisual words (and the face without facial movements) using postproduction video-editing software ( $\mathrm{Ra}$ dius Edit DV, Adobe After-Effects). The soundtrack was then dubbed onto the edited film clips to give a range of audiovisual combinations and recaptured onto two S-VHS videotapes. Each image was shown centered on a high-resolution visual display screen with auditory signals presented at a level of $55 \mathrm{~dB}$. For visual, congruent, and incongruent stimuli, the face remained static for $2 \mathrm{sec}$ before onset of articulation, with each trial lasting $4 \mathrm{sec}$, followed by a 3 -sec blank, during which subjects made their response. For auditory speech stimuli, the face remained static throughout, with onset of the auditory signal $2 \mathrm{sec}$ after visual stimulus onset.

Design. Each session comprised four randomly ordered blocks of the six stimulus words in all eight display types (Upright Unblurred, Upright Blur Level 1, Upright Blur Level 2, Upright Blur Level 3, Inverted Unblurred, Inverted Blur Level 1, Inverted Blur Level 2, and Inverted Blur Level 3) and randomly ordered examples of all four speech conditions (auditory, visual, audiovisual congruent, audiovisual incongruent). Each tape started with eight examples of congruent audiovisual blurred and unblurred upright and inverted display types, shown as practice items.

Procedure. Each participant was seated at a table $1 \mathrm{~m}$ in front of the display screen, with head level with the screen and supported on a chinrest. Participants were instructed to look at the screen and listen throughout the trials and to make their (free-report) responses by writing the word they heard (with the emphasis in the instructions being on heard) on their response sheet. When presented with visual speech trials, participants were instructed to write down the word they thought was being articulated.

\section{Results}

A correct response was recorded whenever a participant wrote down the exact word presented auditorily (auditoryonly and audiovisual speech conditions) or visually (visual speech only condition). Four separate ANOVA's were conducted on data from the auditory-only, visual-only, congruent audiovisual, and incongruent audiovisual speech conditions, with the factors orientation (upright/ inverted), blur level (b0, b1, b2, b3), and stimulus word (/bat/, /din/, /dip/, /map/, /nap/, /vat/). Individual and combined effects of blur, orientation, and their stimulus dependency will be reported separately. 

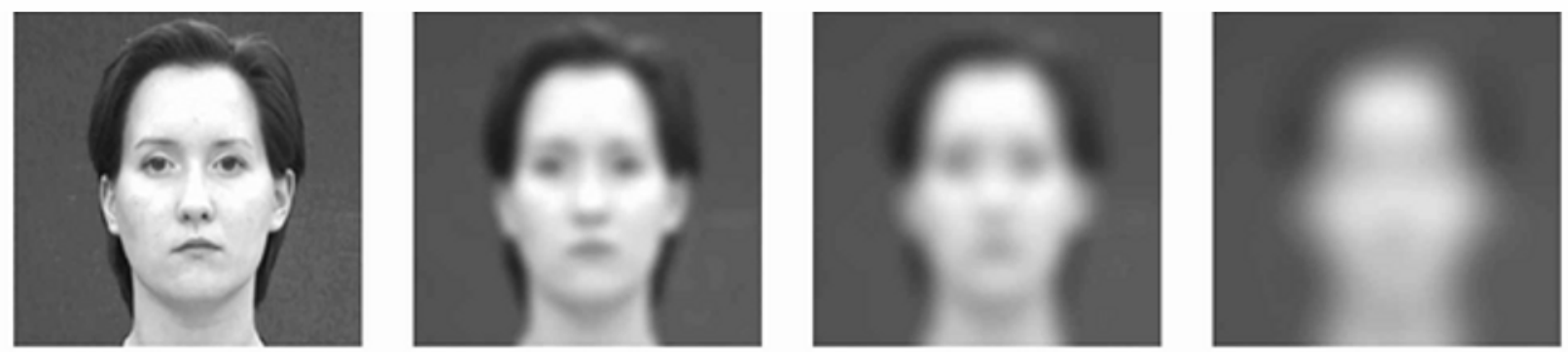

Figure 2. Single-frame examples of the displays used in Experiment 1. One unfiltered and three filtered versions $(288 \mathrm{cycles}$ per face wid th [f/w]; 16 cycles per f/w; 8 cycles per f/w; 4 cycles per f/w). Original displays in color and of DV quality.

Overall auditory performance was $96.4 \%$, and for the auditory condition, there was a main effect of stimulus type $[F(5,45)=3.14, p<.05]$. Post hoc testing (NewmanKeuls) revealed that identification of the word /vat/ was poorer than identification of /dip/,/map/, and /nap/. There were no other main effects or interactions. Thus, the pho- netic identities of all the auditory stimuli used in the experiment were essentially unambiguous and unchanging across display type.

Mean identification accuracy for the visual-only, congruent audiovisual, and incongruent audiovisual conditions across display type is shown in Figure 3. For the sake

\section{Visual Only $\square$ Congruent Audiovisual $\square$ Incongruent Audiovisual}

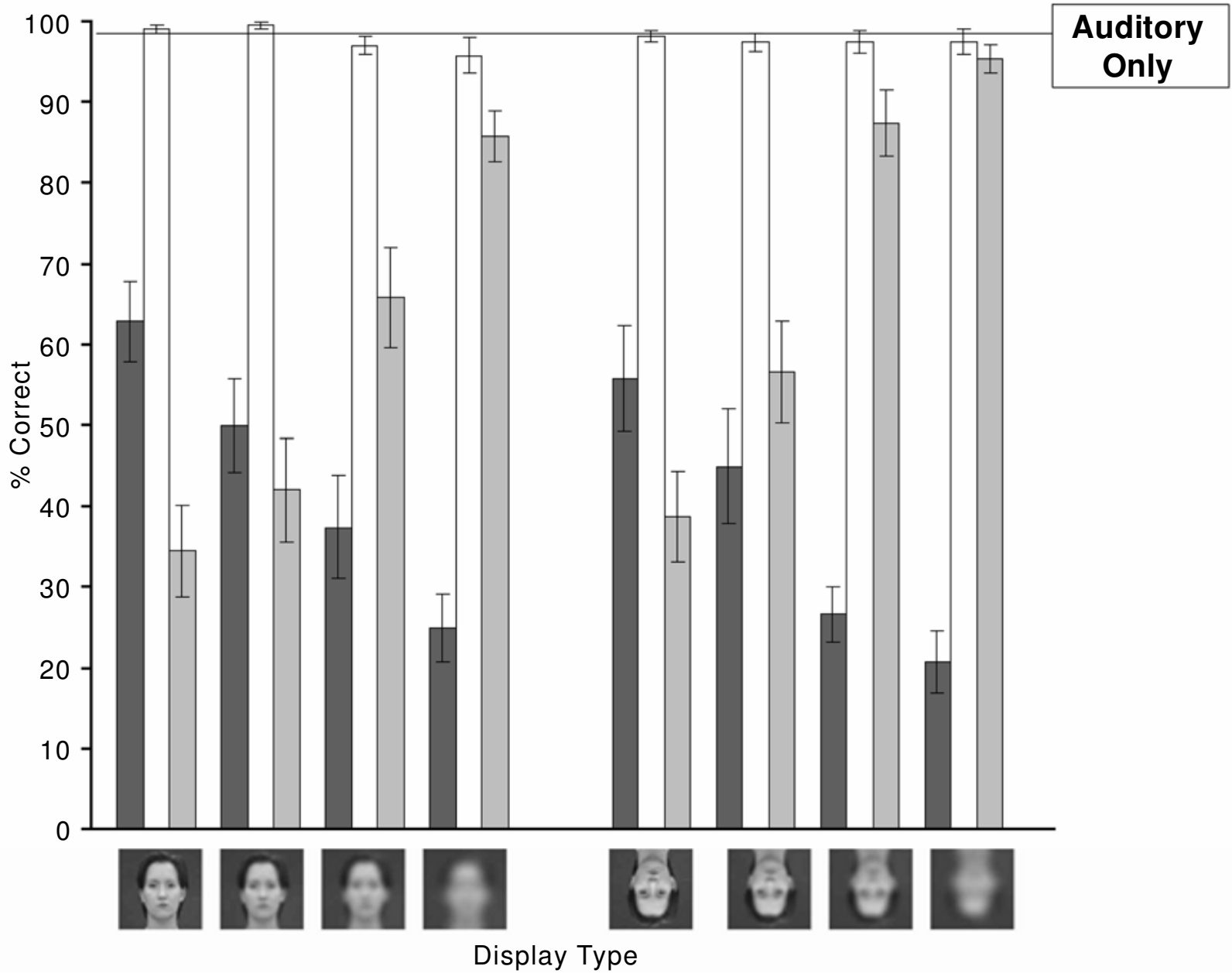

Figure 3. Mean percentage of auditory stimuli correctly identified in upright and inverted visual-only, congruent audiovisual, and incongruent audiovisual displays in Experiment 1 at each blurring level. 
of clarity, only significant effects will be reported, addressing effects of blur, orientation, their combined effects, and stimulus interactions.

Effects of blur. For the visual-only condition, there was a main effect of blur $[F(3,27)=42.51, p<.0001]$, with increasing blur leading to decreasing accuracy (b0 $>$ $\left.\mathrm{b} 1>\mathrm{b} 2, p_{\mathrm{s}}<.01, \mathrm{~b} 2>\mathrm{b} 3, p<.05\right)$. Even at Blur Level 3 , however, visual speech reading performance remained above chance $(16 \%)$.

For congruent audiovisual speech stimuli, there was no main effect of blur. Thus, the accuracy with which congruent audiovisual information was encoded from normal faces remained unaffected when blurred faces were viewed.

For the incongruent audiovisual condition there was a main effect of blur $[F(3,27)=106.66, p<.0001]$. Post hoc testing (Newman-Keuls) revealed significant differences between all four levels of blur, with increasing performance accompanying increasing blur (all $p$ s $<.01$ ).

Effects of orientation. For the visual-only and congruent audiovisual stimuli, no main effects of orientation were observed. For the incongruent audiovisual condition, there was a main effect of orientation $[F(1,9)=99.69$, $p<.0001$ ], with upright faces leading to lower performance than inverted faces.

Combined effects of blur and inversion. For the incongruent audiovisual stimuli, there was an interaction between orientation and blur $[F(3,27)=6.86, p<.01]$, with no effects of inversion for unblurred faces, but significant effects of inversion at Blur Levels 1,2 ( $p$ s $<$ $.001)$, and $3(p<.01)$. Specifically, inversion of the unblurred talking face did not reduce McGurk effects relative to the upright face; however, when the inverted faces were blurred, McGurk effects were much less evident.
Stimulus effects. Mean identification accuracy for individual stimulus words in the visual-only, congruent audiovisual, and incongruent audiovisual conditions across display type is shown in Table 1.

For the visual-only condition, there was an interaction between orientation, blur, and stimulus type $[F(15,135)=$ $2.32, p<.01]$, reflecting an inversion effect for the stimulus /dip/ at Blur Level $1(p<.05)$ and for the stimulus /vat/ at Blur Level $3(p<.01)$.

For the congruent audiovisual stimuli, there was a main effect of stimulus type $[F(5,45)=3.95, p<.01]$. The word /bat/ was identified less accurately than /dip/ and /map/ ( $p$ s < .05), and the stimulus /vat/ was identified less accurately than /dip/, /map/, and /nap/ ( $p$ s $<.05)$.

For the incongruent audiovisual stimuli there was also a main effect of stimulus type $[F(5,45)=9.81, p<$ .0001 ], with incongruent audiovisual pairings /vat//bat/ and /dip//din/ having less effect than all other incongruent pairings $(p s<.05)$.

For the incongruent audiovisual pairings, a three-way interaction between orientation, blur, and stimulus $[F(15,135)=2.39, p<.01]$ reflected significant effects of inversion at Blur Levels 1 and 2 for /bat//vat/ and /din//dip/ $(p \mathrm{~s}<.01)$ and at Blur Level 3 for /bat//vat/ $(p<.001)$.

Comparison of auditory-only and audiovisual speech conditions. In order to assess improvement in auditory identification afforded by congruent visual speech, a comparison was made between the data obtained in the congruent audiovisual condition and the data obtained in the auditory-only condition. An ANOVA with the factors orientation, condition (auditory-only, congruent audiovisual), and blur level revealed no main effects of condition, and condition did not interact with any other factor. This

Table 1

Mean Percentage Correct Responses to Visual and Audiovisual Stimuli Across Displays in Experiment 1

\begin{tabular}{|c|c|c|c|c|c|c|c|c|}
\hline \multirow[b]{2}{*}{ Presentation } & \multicolumn{4}{|c|}{ Upright } & \multicolumn{4}{|c|}{ Inverted } \\
\hline & b0 & b1 & b2 & b3 & b0 & b1 & b2 & b3 \\
\hline \multicolumn{9}{|l|}{ Visual only } \\
\hline /bat/ & 67.5 & 45 & 32.5 & 30 & 55 & 50 & 35 & 15 \\
\hline$/ \operatorname{din} /$ & 52.5 & 37.5 & 27.5 & 15 & 42.5 & 32.5 & 17.5 & 10 \\
\hline /dip/ & 90 & 75 & 42.5 & 35 & 85 & 57.5 & 40 & 35 \\
\hline$/ \mathrm{map} /$ & 52.5 & 52.5 & 47.5 & 17.5 & 65 & 40 & 35 & 22.5 \\
\hline /nap/ & 57.5 & 40 & 32.5 & 25 & 37.5 & 40 & 20 & 22.5 \\
\hline /vat/ & 57.5 & 50 & 42.5 & 27.5 & 50 & 50 & 12.5 & 20 \\
\hline \multicolumn{9}{|c|}{ Congruent audiovisual } \\
\hline /bat/ & 100 & 97.5 & 90 & 90 & 97.5 & 95 & 97.5 & 97.5 \\
\hline$/ \operatorname{din} /$ & 100 & 100 & 95 & 95 & 100 & 100 & 95 & 95 \\
\hline /dip/ & 100 & 100 & 100 & 97.5 & 100 & 100 & 100 & 100 \\
\hline$/ \mathrm{map} /$ & 100 & 100 & 97.5 & 100 & 100 & 97.5 & 100 & 100 \\
\hline /nap/ & 100 & 100 & 100 & 97.5 & 100 & 100 & 97.5 & 97.5 \\
\hline /vat/ & 95 & 100 & 100 & 95 & 92.5 & 92.5 & 95 & 95 \\
\hline \multicolumn{9}{|c|}{ Incongruent audiovisual } \\
\hline /bat//vat/ & 0 & 10 & 57.5 & 67.5 & 2.5 & 32.5 & 95 & 97.5 \\
\hline /din//dip/ & 2.5 & 12.5 & 35 & 72.5 & 7.5 & 30 & 85 & 92.5 \\
\hline /dip//din/ & 77.5 & 77.5 & 90 & 100 & 72.5 & 87.5 & 90 & 100 \\
\hline$/ \mathrm{map} / / \mathrm{nap} /$ & 32.5 & 42.5 & 75 & 100 & 32.5 & 50 & 82.5 & 97.5 \\
\hline$/ \mathrm{nap} / / \mathrm{map} /$ & 37.5 & 42.5 & 57.5 & 77.5 & 47.5 & 50 & 77.5 & 90 \\
\hline /vat//bat/ & 57.5 & 67.5 & 80 & 97.5 & 70 & 80 & 95 & 95 \\
\hline
\end{tabular}

Note-b0, Unblurred; b1, Blur Level 1; b2, Blur Level 2; b3, Blur Level 3. 
result is unsurprising given the high levels of performance in the auditory-only condition. We will return to this point in Experiment 2.

In order to assess decrements in auditory identification afforded by congruent visual speech, the data obtained in the incongruent audiovisual condition were also compared with the data obtained in the auditory-only condition. There was a main effect of condition $[F(1,9)=108.55$, $p<.0001$ ], with lower accuracy in the incongruent audiovisual speech condition relative to the auditory-only condition. In the incongruent audiovisual speech condition, there was also a four-way interaction between all the factors $[F(15,135)=1.89, p<.05]$. For the incongruent audiovisual pairings /bat//vat/ and /din//dip/, there were decrements in performance relative to the auditory-only condition for every display apart from Inverted Blur Levels 2 and 3 .

For the incongruent audiovisual stimuli /map//nap/ and /nap//map/, there were decrements in performance for the incongruent audiovisual condition relative to the auditoryonly condition for every display apart from Inverted Blur Level 2 and all Blur Level 3 displays.

The incongruent audiovisual stimuli /vat//bat/ and /dip//din/ were comparatively ineffective overall, as evidenced by decrements in performance relative to the auditory-only condition for unblurred and Blur Level 1 upright displays of /vat//bat/, and unblurred displays of /dip//din/.

Thus, the influence of incongruent visual speech on perception of the auditory signal was most evident with upright and inverted faces that were unblurred or displayed at Blur Level 1. It dissipated, however, for Inverted Blur Levels 2 and 3 displays.

In summary, Experiment 1 demonstrated effects of inversion for the visual-only stimulus /dip/ at Blur Level 1 and for the visual-only stimulus /vat/ at Blur Level 3. There were effects of inversion for the incongruent audiovisual stimulus item /bat//vat/ at Blur Levels 1,2, and 3, for the stimulus item/din//dip/at Blur Levels 1 and 2, and for the stimulus item /vat//bat/ at Blur Level 1. Most importantly, there were no inversion effects for any stimulus when the face was unblurred. Incongruent visual speech was equally effective at altering the perceived speech sound when faces were inverted as when they were upright. However, when the face was blurred, inversion effects were observed, especially for the stimulus item /bat//vat/.

\section{Discussion}

The findings of Experiment 1 indicate that the processes responsible for encoding facial movement information tolerate substantial degradation of visible facial information. Perception of visual speech per se, and the influence of visual speech on perceived speech sounds, is robust even when featural detail is removed by blurring. Accurate visual speech identification perception was evident down to 8 cycles per $\mathrm{f} / \mathrm{w}$ displays, and the influence of incongruent visual speech on auditory speech perception was evident down to 4 cycles per f/w displays. As indicated by the pilot study, it appears that perception of configural relations conveyed in blurred talking faces is sufficient to convey visual speech information. Performance with visual speech per se and effects of visual speech on auditory speech perception do, however, decrease with blurring, implying that higher spatial frequencies are carrying some information about visual speech.

Contrary to previous results (e.g., Jordan \& Bevan, 1997; Rosenblum et al., 2000), inversion of unblurred talking faces did not affect the influence of visual speech on perceived speech sounds. It is possible that this discrepancy occurred because of differences in the individual speakers used in different experiments, or that the stimulus displays differed in clarity/availability of visual detail. In addition, the present experiments differ from previous investigations in that the stimulus items were monosyllabic CVC words rather than CV tokens (Green, 1994; Jordan \& Bevan, 1997; Massaro \& Cohen, 1996, Rosenblum et al., 2000) or VCV tokens (Bertelson, Vroomen, Wiegeraad, \& de Gelder, 1994). The visual speech information conveyed by articulating these words is perhaps more comprehensive than that conveyed by $\mathrm{CV}$ syllables, and therefore more detectable even when faces are inverted. Specifically, all the tokens had distinct visual beginnings and endings-for example, $/ \mathrm{n} /$ and $/ \mathrm{p} /$ for $/ \mathrm{nap} /$. This extra information may have reduced any potential disruption caused by inversion.

More importantly in the present experiments, however, blurring inverted talking faces reduced the influence of visual speech on perceived speech sounds. This finding may suggest that fine visual information is used by observers when they are attempting to encode visual speech from inverted faces. Reliance on featural detail when faces are inverted is supported in part by the nature of the inversion effects obtained across different stimuli. For example, the incongruent audiovisual pairing / bat//vat/ led to equal levels of McGurk effects inverted as upright when the stimulus items were unblurred. Here, observers may have utilized fine information such as the appearance of the teeth over the lower lip (albeit upside-down) to influence what they heard. When this information became blurred, however, observers were much less susceptible to this influence. This is supported by the finding of an inversion effect for /vat/ in the visual-only condition at Blur Level 3.

Similarly, the stimulus item/din//dip/ may have suffered from blurring the relatively fine lip movements used in articulating /dip/. Indeed, /dip/ involved much finer movement relative to the other stimuli, with lip separation of only around 8 pixels, compared with, for example, $/ \mathrm{map} /$, where lip separation was on the order of 35 pixels. No inversion effects were obtained with the stimulus items /map//nap/, /nap//map/, or/dip//din/. The stimulus item/dip//din/ was quite ineffective overall, but the items /map//nap/ and /nap//map/ may have been equally detectable in upright and inverted faces because of the coarse visual cues by which they are characterized (i.e., a broad vertical mouth opening, with a bilabial closure). The implication from the present results is that when faces are inverted, accurate visual speech information may still be extracted because of 
support afforded by coarse visual cues, such as bilabial separations and the direction of facial motion, and detailed visual cues where appropriate. However, when visual detail is removed from the inverted faces by blurring, reliance must be on the coarse visual information, which preserves the influence of stimuli like /map/, but does not preserve the influence of articulations specified by finer detail such as /vat/ and /dip/.

However, before fully exploring the implications of the present results, we need to ensure that ceiling effects in perception of the auditory signal in Experiment 1 did not hide any potential performance improvements or decrements in the audiovisual conditions. A great deal of research suggests that the influence of visual speech on auditory speech recognition increases when the signal-tonoise ratio of the auditory signal decreases (e.g., Jordan \& Sergeant, 1998; MacLeod \& Summerfield, 1987, 1990; Middleweerd \& Plomp, 1987; Sumby \& Pollack, 1954). For example, Jordan and Sergeant (1998) found that although manipulating the quality of visual speech (in this case, by changing the size of facial images) produced changes in the influence of visual speech on congruent and incongruent auditory speech recognition in a noise-free environment, these effects were more widespread and generally more substantial when auditory signals were presented in a background of white noise. In a similar vein, presenting auditory signals in white noise may provide a more sensitive test of changes in visual speech influence across orientation and blur, especially in the congruent audiovisual condition, where there was little room for improvement over auditory-only performance in Experiment 1. Thus, in Experiment 2, auditory signals were presented in a background of white noise in order to provide appropriate "headroom" for revealing individual effects of display type on the influences exerted by congruent (and incongruent) visual speech.

\section{EXPERIMENT 2}

In Experiment 2 we investigated the influence of inversion and blurring on the perception of visual and audiovisual speech.

\section{Method}

The same words were used as in Experiment 1 in their visual-only, auditory-only, congruent, and incongruent forms. However, these stimuli were now presented in a background of continuous white noise at a sound level of $70 \mathrm{~dB}$. Pretesting had established that a signal-tonoise ratio of $-15 \mathrm{~dB}$ produced approximately $50 \%$ correct responses to auditory stimuli presented without any visual speech. This midpoint of the performance scale was chosen to provide "room" for visual speech influences. The white noise was presented via a separate loudspeaker placed adjacent to both signal speakers and the visual display. All remaining aspects of this experiment were the same as in Experiment 1. No participants in Experiment 2 had engaged in Experiment 1.

\section{Results}

ANOVAs were conducted on data from the four different conditions - auditory-only, visual-only, congruent au- diovisual, and incongruent audiovisual speech. The addition of white noise brought auditory performance down to $36 \%$. An ANOVA conducted on the data from the auditoryonly condition revealed a main effect of stimulus $[F(5,45)=$ $13.05, p<.0001]$. Identification of /nap/ was more accurate than that for any other stimulus ( $p$ s $<.01)$, and identification of /map/ was better than all except/nap/ $(p s<.05)$. There were no other main effects or interactions. Thus, the phonetic identities of all the auditory stimuli used in the experiment were unchanging across display type.

Mean identificationaccuracy for the visual-only, congruent audiovisual, and incongruent audiovisual conditions across display type is shown in Figure 4. Again, only significant effects will be reported, addressing effects of blur and orientation, and their combined effects and stimulus interactions.

Effects of blur. The ANOVA for the visual-only condition revealed a main effect of blur $[F(3,27)=38.83, p<$ $.0001]$, reflecting decreasing accuracy with increasing blur (b0 $>$ b1 $>$ b2, $p$ s $<.01,>b 3, p<.05$ ). For the congruent audiovisual speech condition, there was also a main effect of blur $[F(3,27)=63.58, p<.0001]$, reflecting decreasing accuracy with increasing blur, b0 $>$ b1 $>$ $\mathrm{b} 2>\mathrm{b} 3(p \mathrm{~s}<.01)$. For the incongruent audiovisual speech condition, there was also a main effect of blur $[F(3,27)=$ $39.17, p<.0001]$. Post hoc testing (Newman-Keuls) revealed significant differences between blur levels, with $\mathrm{b} 0=\mathrm{b} 1<\mathrm{b} 2<\mathrm{b} 3($ all $p \mathrm{~s}<.001)$.

Effects of orientation. For the congruent audiovisual speech condition, there was a main effect of orientation $[F(1,9)=20.27, p<.01]$, reflecting greater accuracy in the upright compared with the inverted displays. In the incongruent audiovisual condition, there was also a main effect of orientation $[F(1,9)=6.45, p<.05]$, with upright faces leading to lower performance than inverted faces.

Combined effects of blur and orientation. For the congruent audiovisual stimuli there was an interaction between orientation and blur $[F(3,27)=3.13, p<.05]$, reflecting inversion effects at Blur Levels 1 and $2(p$ s $<$ $.01)$. For the incongruent audiovisual stimuli there was also an interaction between orientation and blur $[F(3,27)=$ $5.83, p<.01]$, with no effects of inversion for unblurred faces, but significant effects of inversion at Blur Levels 1 , $(p<.05), 2(p<.001)$, and $3(p<.05)$. As was the case in Experiment 1, inversion of the unblurred talking face did not reduce McGurk effects relative to the upright; however, when the inverted faces were blurred, McGurk effects were much less evident.

Effects of stimulus type. Mean identification accuracy for individual stimulus words in the visual-only, congruent audiovisual, and incongruent audiovisual conditions across display type is shown in Table 2.

For the visual-only stimuli, there was a main effect of stimulus type $[F(5,45)=5.73, p<.0001]$, reflecting lower accuracy for the stimulus /din/ relative to /bat/, /map/ ( $p$ s $<.01)$, and /nap/ $(p<.05)$, and lower accuracy for the stimulus /vat/ relative to /bat/ and /map/ $(p<.01)$. There was also an interaction between blur level and stim- 


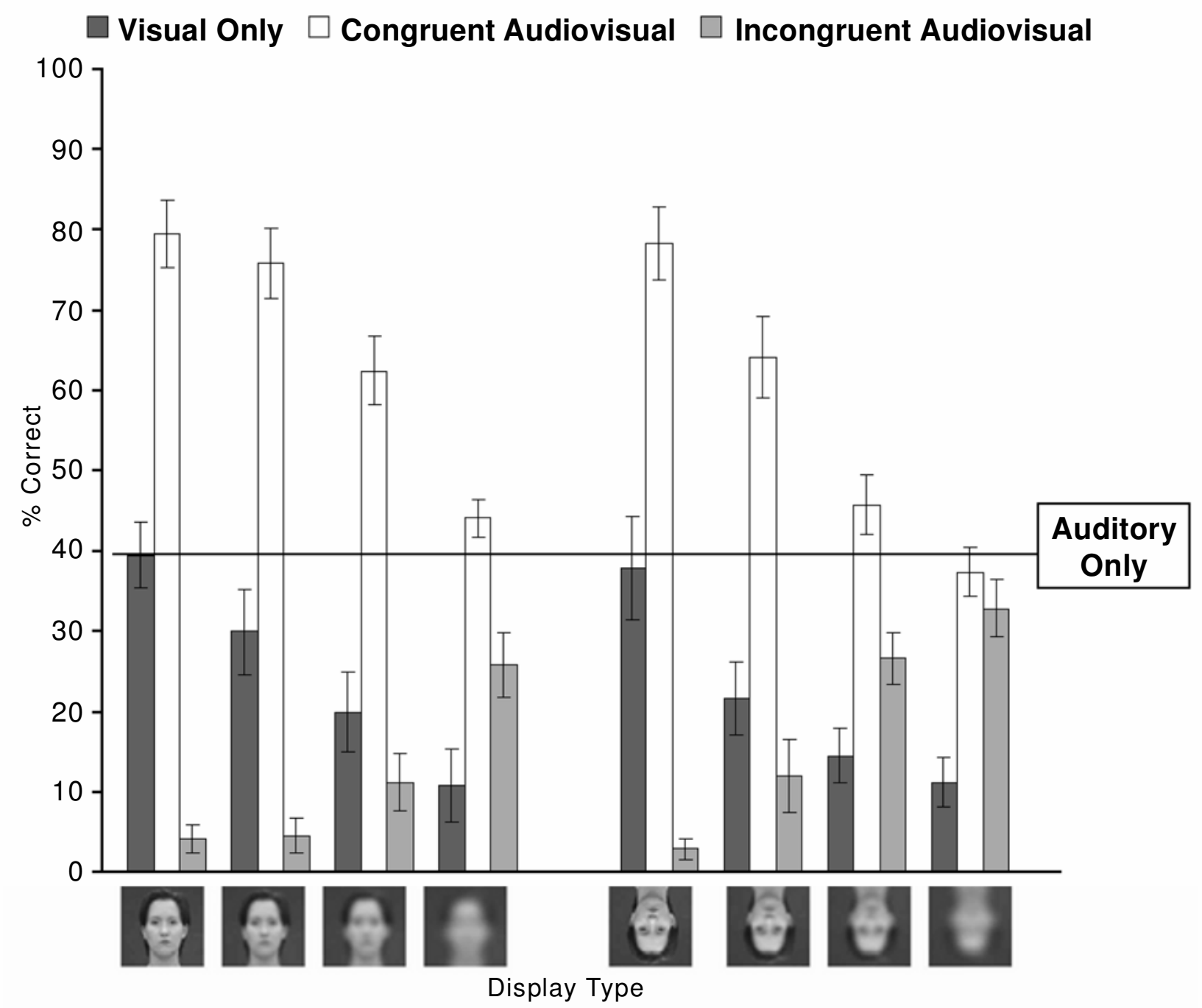

Figure 4. Mean percentage of auditory stimuli correctly identified in upright and inverted visual-only, congruent audiovisual, and incongruent audiovisual displays in Experiment 2 at each blurring level.

ulus type $[F(15,135)=3.62, p<.0001]$, reflecting decreasing accuracy from blur levels $(\mathrm{b} 0>\mathrm{b} 1>\mathrm{b} 2)$ for the stimulus /bat/ $(p \mathrm{~s}<.05)$.

For the congruent audiovisual stimuli there was also a main effect of stimulus type $[F(5,45)=6.99, p<.0001]$. The stimulus/nap/ was identified more accurately than any other stimulus (/din/ and /dip/, $p \mathrm{~s}<.01 ; / \mathrm{map} /$ and $/ \mathrm{vat} /$, $p s<.05)$, and the stimulus /map/ was identified more accurately than $/ \mathrm{din} /$ or $/ \mathrm{dip} /($ all $p \mathrm{~s}<.01)$. An interaction between orientation and stimulus type $[F(5,45)=5.81$, $p<.001]$ reflected inversion effects for the stimuli $/ \mathrm{map} /$ $(p<.05)$ and $/ \mathrm{vat} /(p<.001)$. An interaction between blur and stimulus type $[F(15,135)=2.99, p<.001]$ reflected a decrease in accuracy from Blur Level 1 to Blur Level 2 for $/ \mathrm{bat} /$ and $/ \mathrm{vat} /(p \mathrm{~s}<.001)$, and between Blur Level 2 and Blur Level 3 for $/ \mathrm{map} /(p<.05)$. There was also a three-way interaction between orientation, blur, and stimulus $[F(15,135)=5.81, p<.01]$, reflecting inversion effects at Blur Levels 2 and 3 for the stimulus /vat/ ( $p$ s $<$ $.01)$. Thus, reducing perception of the auditory signal revealed inversion effects with congruent blurred facial movements, though not with unblurred facial movements.

For the incongruent audiovisual condition, an interaction between blurring and stimulus $[F(15,135)=5.93, p<$ $.0001]$ reflected increases in performance with increasing levels of blur for incongruent audiovisual pairings/bat//vat/ (b0 = b1 < b2, $p<.01=$ b3), /map//nap/ (b0 = b1 $<$ $\mathrm{b} 2<\mathrm{b} 3, p \mathrm{~s}<.001)$, and $/ \mathrm{nap} / / \mathrm{map} /(\mathrm{b} 0=\mathrm{b} 1=\mathrm{b} 2<\mathrm{b} 3$, $p<.001)$. A three-way interaction between orientation, blur, and stimulus $[F(15,135)=1.89, p<.05]$ reflected no inversion effects for unblurred faces in any of the incongruent audiovisual pairings, but significant effects of inversion at Blur Level $2 \mathrm{for} / \mathrm{bat} / \mathrm{vat} / \mathrm{and} / \mathrm{map} / \mathrm{nap} /\left(p_{\mathrm{s}}<\right.$ $.01)$ and at Blur Level 3 for /bat//vat/ $(p<.001)$. 
Table 2

Mean Percentage Correct Responses to Visual and Audiovisual Stimuli Across Displays in Experiment 2

\begin{tabular}{|c|c|c|c|c|c|c|c|c|}
\hline \multirow[b]{2}{*}{ Presentation } & \multicolumn{4}{|c|}{ Upright } & \multicolumn{4}{|c|}{ Inverted } \\
\hline & b0 & b1 & $\mathrm{b} 2$ & b3 & b0 & b1 & b2 & b3 \\
\hline \multicolumn{9}{|l|}{ Visual only } \\
\hline /bat/ & 75 & 50 & 27.5 & 10 & 55 & 47.5 & 22.5 & 7.5 \\
\hline$/ \operatorname{din} /$ & 20 & 22.5 & 2.5 & 2.5 & 25 & 0 & 5 & 2.5 \\
\hline /dip/ & 42.5 & 22.5 & 17.5 & 15 & 32.5 & 17.5 & 15 & 10 \\
\hline /map/ & 50 & 45 & 35 & 7.5 & 57.5 & 30 & 30 & 15 \\
\hline /nap/ & 42.5 & 32.5 & 22.5 & 17.5 & 45 & 25 & 12.5 & 22.5 \\
\hline /vat/ & 7.5 & 7.5 & 15 & 12.5 & 10 & 10 & 2.5 & 10 \\
\hline \multicolumn{9}{|c|}{ Congruent audiovisual } \\
\hline /bat/ & 87.5 & 87.5 & 55 & 30 & 82.5 & 77.5 & 40 & 30 \\
\hline /din/ & 65 & 52.5 & 27.5 & 25 & 55 & 40 & 37.5 & 32.5 \\
\hline /dip/ & 55 & 47.5 & 37.5 & 25 & 60 & 45 & 42.5 & 27.5 \\
\hline /map/ & 95 & 90 & 92.5 & 67.5 & 90 & 72.5 & 75 & 50 \\
\hline /nap/ & 95 & 90 & 92.5 & 72.5 & 92.5 & 90 & 75 & 75 \\
\hline /vat/ & 80 & 87.5 & 70 & 45 & 90 & 60 & 5 & 10 \\
\hline \multicolumn{9}{|c|}{ Incongruent audiovisual } \\
\hline /bat//vat/ & 0 & 0 & 15 & 7.5 & 0 & 15 & 45 & 42.5 \\
\hline /din//dip/ & 0 & 0 & 7.5 & 5 & 0 & 10 & 15 & 17.5 \\
\hline /dip//din/ & 12.5 & 15 & 15 & 22.5 & 2.5 & 17.5 & 20 & 17.5 \\
\hline /map//nap/ & 2.5 & 0 & 20 & 60 & 0 & 12.5 & 47.5 & 57.5 \\
\hline$/ \mathrm{nap} / / \mathrm{map} /$ & 5 & 5 & 2.5 & 25 & 5 & 5 & 12.5 & 42.5 \\
\hline /vat//bat/ & 5 & 7.5 & 7.5 & 25 & 10 & 12.5 & 20 & 20 \\
\hline
\end{tabular}

Note-b0, Unblurred; b1, Blur Level 1; b2, Blur Level 2; b3, Blur Level 3.

Comparison of auditory-only and audiovisual speech conditions. A comparison was made between the data obtained in the congruent audiovisual condition and the data obtained in the auditory-only condition. An ANOVA with the factors orientation, condition (auditory-only, congruent audiovisual), and blur level revealed a main effect of condition $[F(1,9)=112.36, p<.0001]$, with increased accuracy in the congruent audiovisual speech condition relative to the auditory-only condition. There was also a four-way interaction between all the factors $[F(15,135)=$ $2.33, p<.01]$. For upright and inverted displays of /bat/ there were increases in performance compared with the auditory-only condition for unblurred and Blur Level 1 displays, and also for Upright Blur Level 2 displays. For $/ \mathrm{map} /$ and /vat/ there were increases in performance for unblurred upright and inverted displays, and Upright Blur Level 1 and 2 displays. For /din/ there were increases in performance for unblurred and Blur Level 1 upright faces. For /dip/ there were increases in performance only for unblurred upright and inverted displays. For/nap/there were increases in unblurred upright displays. Thus the improving effects of congruent visual speech on auditory perception were observed for unblurred displays and became less effective when faces were inverted and blurred.

The data obtained in the incongruent audiovisual condition were compared with the data obtained in the auditoryonly condition. An ANOVA revealed a main effect of condition $[F(1,9)=79.31, p<.0001]$, with significantly lower accuracy in the incongruent audiovisual speech condition relative to the auditory-only condition.

An interaction between condition, orientation, and stimulus $[F(5,45)=2.78, p<.05]$ reflected decrements in performance for the incongruent audiovisual condition relative to the auditory-only condition for upright $/ \mathrm{map} / / \mathrm{nap} /$ and $/ \mathrm{nap} / / \mathrm{map} /(p \mathrm{~s}<.001)$, and inverted $/ \mathrm{din} / / \mathrm{dip} /(p<.05), / \mathrm{map} / / \mathrm{nap} /$, and $/ \mathrm{nap} / / \mathrm{map} /(p \mathrm{~s}<$ $.001)$. An interaction between condition, blur, and stimulus $[F(15,135)=4.05, p<.0001]$ reflected decrements in performance for the incongruent audiovisual condition relative to the auditory-only condition for all displays of /nap//map/, unblurred, and Blur Level 1 and 2 displays of /map//nap/ and at unblurred displays of /din//dip/.

In summary, there were no effects of inversion for normal unblurred displays. Congruent and incongruent visual speech was as effective when faces were inverted as when they were upright. However, when the face was blurred, inversion effects were observed, particularly with congruent /vat/ and incongruent/bat//vat/.

\section{Discussion}

The findings of Experiment 2 confirmed results from Experiment 1 in that the processes responsible for encoding facial movement information tolerate substantial degradation of visible facial information. There were no effects of inversion for unblurred talking faces, but as in Experiment 1, inversion of blurred talking faces reduced the influence of incongruent visual speech on perceived speech sounds. In addition, when presented in auditory noise, inversion of blurred talking faces reduced the beneficial influence of congruent visual speech on perceived speech sounds.

Stimulus effects in Experiment 2 were similar to those observed in Experiment 1. Again, the incongruent audiovisual pairing /bat//vat/ led to equal levels of McGurk ef- 
fects inverted as upright when the stimulus items were unblurred, but blurring introduced inversion effects. Moreover, when presented in auditory noise, inversion effects were observed for the congruent audiovisual/vat/. As suggested previously, observers may have utilized the appearance of the teeth over the lower lip to influence what they heard. When this information became blurred, however, observers were much less susceptible to this influence.

Unlike Experiment 1, this experiment yielded no inversion effects with the stimulusitem/din//dip/. This appears to be due to floor effects in this case, since the illusion was stronger when presented in auditory noise, and even the very blurred inverted faces produced McGurk effects. The addition of auditory noise in Experiment 2 also revealed an inversion effect for the stimulus /map//nap/. In line with our previous interpretations of the effects in terms of availability of detailed visual information, reliance on the detailed cue of the alveolar/n/ (where tongue visibility is important) may have been disrupted by blurring.

\section{GENERAL DISCUSSION}

In the experiments reported in this article we investigated the separate and combined effects of facial orientation and blurring on the identification of visual speech per se and on the influence of visual speech on the recognition of congruent and incongruent auditory speech. The main findings were as follows: (1) Blurring affected report of visual speech in quiet, although performance remained well above chance down to 8 cycles per f/w display. (2) Blurring did not affect the influence of visual speech on report of congruent auditory speech in quiet. When presented in a background of white noise, blurring reduced perception of the congruent auditory signal, although perception of the auditory signal was improved by the addition of congruent visual speech down to 8 cycles per f/w display. (3) Blurring affected the influence of incongruent visual speech on perception of the auditory signal in quiet and in noise, although this influence remained down to 8 cycles per f/w display. (4) Inversion of normal unblurred faces did not affect perception of visual speech per se, the influence of visual speech on report of congruent auditory speech in quiet and in noise, or the influence of incongruent visual speech on perception of the auditory signal in quiet and in noise. (5) Inversion of blurred faces affected perception of the visual speech signal in quiet, affected the influence of incongruent visual speech on the auditory signal in quiet and noise, and affected the influence of congruent speech on auditory speech perception in noise. These inversion effects were dependent on the stimulus tokens displayed.

The first point to emerge from these findings is that performance with visual and audiovisual speech stimuli shows considerable resilience to reduction in facial detail. Second, performance with visual and audiovisual speech stimuli shows considerable resilience to facial inversion. Jordan and Bevan (1997) reported inversion effects only when much of the visual information in upright faces was preserved in inverted faces, as with visual /ga/. In the present experiments, no inversion effects emerged when the displays were unblurred. As we pointed out previously, this finding is discrepant with previous investigations, and may have occurred because of differences in the individual speakers used in different experiments, differences in the visual displays used, or the use of monosyllabic CVC words rather than the CV tokens (Green, 1994; Jordan \& Bevan, 1997; Massaro \& Cohen, 1996; Rosenblum et al., 2000) or VCV tokens (Bertelson et al., 1994) employed in previous investigations. The visual speech information conveyed by articulating these words is perhaps more comprehensive than that conveyed by CV syllables.

This insensitivity of the visual and audiovisual speech system to facial inversion indicates that the visible characteristics used to distinguish speech movements in upright faces were equally well encoded in inverted faces. This insensitivity to facial orientation can be reasonably explained by the notion that sufficient information about the identity of speech movements can be provided by certain basic cues (such as overall mouth shape and the nature and timing of bilabial movements; e.g., Walden, Prosek, Montgomery, Scherr, \& Jones, 1977) that are not degraded by facial inversion. Thus, for example, when the face movements for /map/ are presented, a substantial cue to the identity of the word may be that a bilabial closure is present at the beginning and end of the utterance. Basic cues such as this may be equally apparent in inverted as in upright faces, and , moreover, may be equally apparent in clear and blurred faces. However, as we have demonstrated, this is not the case for all face movements. Labiodental and linguodental tokens such as /v/ and /n/ may be equally apparent in upright and inverted faces, but not in blurred inverted faces. The systematic manipulation of a wide range of phonemic variables representing varying levels of movement, as well as teeth and tongue conspicuity, is a topic for future research and may provide valuable clues to the causes of variable results hitherto observed in experiments that address the inversion of talking faces.

The finding that blurring produced an inversion effect suggests that facial blurring induced a change in the processing of facial speech movement information. Specifically, when talking faces are upright, both coarse configural and fine featural information may be used by observers. When faces are inverted, configural information is disrupted, leading to a reliance on fine featural information. When perception of this fine information is impaired by blurring, inversion effects emerge that are similar to those reported for facial recognition. The detrimental effects of inversion on face perception were not mirrored in the present results for unfiltered talking faces. As we have already pointed out (see introduction), numerous studies have shown that facial inversion produces impairments in observers' ability to recognize faces. Moreover, this impairment has inspired the popular notion that face recognition is dependent on relational features between the various parts of an upright face so that when faces are inverted, these re- 
lational features become difficult to encode and face recognition performance deteriorates. Thus, face recognition may rely more heavily on the perception of structural configural relations present in upright faces than does visual and audiovisual speech perception. As is evident from the present results with blurred faces, the visual and audiovisual speech processing system can use coarse information when it is all that is available, but it may not normally be the dominant determinant of accurate audiovisual speech perception. This role may generally fall to more detailed visual information, which effectively survives inversion. However, the finding of inversion effects with blurred faces does suggest that some of the information used in visual and audiovisual speech perception is dependent on an upright facial context, a result that complements previous findings suggesting a commonality with face perception (e.g., Jordan \& Bevan, 1997; Rosenblum et al., 2000). We suggest that the resilience to inversion found with unblurred faces in the present experiments is founded on the detection of visually fine aspects of the talking face, aspects that may play a smaller role in face recognition. This is supported by the finding that blurring the talking face results in inversion effects. Here, perception of fine detail is severely impaired, and, contrary to the case with upright faces, the use of configural information to withstand this impairment is not possible, resulting in inversion effects commonly observed in studies of face recognition. Specifically, we suggest that when perception of precise visual motion and detail is possible, observers may use this information to identify visual and audiovisual speech. When perception of precise visual detail is impaired, as when the stimulus is blurred or seen from a distance, observers can use coarse configural information to identify visual and audiovisual speech. When perception of precise visual detail and coarse configural information is impaired, identification of visual and audiovisual speech is adversely affected. Such putative shifts in encoding strategy require further investigation, but it is possible that the representations for visual speech have thresholds that are variable according to the visual input available. In general, however, to be met, these thresholds require little information. Even inverted, very blurred facial movements can have an influence on what we hear. This power to transcend visual variation within talking faces has doubtless come about through the ecological importance of understanding speech in everyday communications and complements the growing picture of a highly robust and adaptable visual and audiovisual speech recognition system.

\section{REFERENCES}

Bachmann, T. (1991). Identification of spatially quantized tachistoscopic images of faces: How many pixels does it take to carry identity? European Journal of Cognitive Psychology, 3, 87-103.

BAsSILI, J. N. (1978). Facial motion in the perception of faces and of emotional expression. Journal of Experimental Psychology: Human Perception \& Performance, 4, 373-379.

Berry, D. S. (1990). What can a moving face tell us? Journal of Personality \& Social Psychology, 58, 1004-1014.

Bertelson, P., Vroomen, J., Wiegeraad, G., \& de Gelder, B. (1994).
Exploring the relation between McGurk interference and ventriloquism. Proceedings of the 1994 International Conference on Spoken Language Processing (ICLP94), 2, 559-562.

BingHAM, G. P. (1987). Scaling and kinematic form: Further investigations on the visual perception of lifted weight. Journal of Experimental Psychology: Human Perception \& Performance, 13, 155-177.

Brookes N. M., \& Templeton, P. D. (1990). Visual speech intelligibility of digitally processed facial images. Proceedings of the Institute of Acoustics, 12, 483-490.

Campbell, C. S., \& Massaro, D. W. (1997). Perception of visible speech: Influence of spatial quantization. Perception, 26, 627-644.

Carey, S., \& Diamond, R. (1977). From piecemeal to configurational representation of faces. Science, 195, 312-314.

Costen, N. P., Parker, D. M., \& Craw, I. (1996). Effects of high-pass and low-pass spatial filtering on face identification. Perception \& Psychophysics, 58, 602-612.

De Valois, R. L., \& De VAlois, K. K. (1988). Spatial vision. New York: Oxford University Press.

ERBER, N. P. (1969). Interaction of audition and vision in the recognition of oral speech stimuli. Journal of Speech \& Hearing Research, 12, 423-425.

Frowein, H. W., Smoorenburg, G. F., Pyters, L., \& Schinkel, D. (1991). Improved speech recognition throughtelephony: Experiments with the hard of hearing. IEEE Journal on Selected Areas in Communications, 9, 611-616.

Green, K. P. (1994, May). The influence of an inverted face on the McGurk effect. Poster presented at the meeting of the Acoustical Society of America, Cambridge, MA.

JohANSSON, G. (1973). Visual perception of biological motion and a model for its analysis. Perception \& Psychophysics, 14, 201-211.

Johnson, J. A., Rosenblum, L. D., \& Saldaña, H. M. (1994). The contribution of a reduced visual image to speech perception in noise [Abstract]. Poster presented at the 127th meeting of the Acoustical Society of America. Journal of the Acoustical Society of America, 95, 3009.

Jordan, T. R, \& BeVAn, K. [M.] (1997). Seeing and hearing rotated faces: Influences of facial orientation on visual and audiovisual speech recognition. Journal of Experimental Psychology: Human Perception \& Performance, 23, 388-403.

Jordan, T. R., McCotter, M. V., \& Thomas, S. M. (2000). Visual and audiovisual speech perception with color and gray scale facial images. Perception \& Psychophysics, 62, 1394-1404.

Jordan, T. R, \& SERgeant, P. C. (1998). Effects of facial image size on visual and audio visual speech recognition. In R. Campbell, B. Dodd, \& D. Burnham (Eds.), Hearing by eye II: Advances in the psychology of speechreading and auditory-visual speech (pp. 155-176). Hove, U.K.: Psychology Press.

Jordan, T. R., \& SERgEAnt, P. [C.] (2000). Effects of distance on visual and audiovisual speech recognition. Language \& Speech, 43, 107-124.

JoRDAN, T. R., \& THOMAs, S. M. (2001). Effects of horizontal viewing angle on visual and audiovisual speech recognition. Journal of Experimental Psychology: Human Perception \& Performance, 27, 13861403.

Kanzaki, R., Kato, T., \& TohKura, Y. (1998). Influence of facial views on the McGurk effect. Journal of the Acoustical Society of Japan, 19, 69-72.

Kozwolski, L. T., \& Cutting, J. E. (1977). Recognizing the sex of a walker from a dynamic point-light display. Perception \& Psychophysics, 21, 575-580.

Leder, H., \& BrUce, V. (2000). When inverted faces are recognized: The role of configural; information in face recognition. Quarterly Journal of Experimental Psychology, 53A, 513-536.

Liberman, A. M., \& Mattingly, I. G. (1985). The motor theory of speech perception revised. Cognition, 21, 1-36.

MacLeod, A., \& Summerfield, Q. (1987). Quantifying the contribution of vision to speech perception in noise. British Journal of Audiology, 12, 131-141.

MacLeod, A., \& Summerfield, Q. (1990). A procedure for measuring auditory and audio-visual speech-reception thresholds for sentences in noise: Rationale, evaluation, and recommendations for use. British Journal of Audiology, 24, 29-43. 
Massaro, D. W., \& Cohen, M. M. (1996). Perceiving speech from inverted faces. Perception \& Psychophysics, 58, 1047-1065.

McGurK, H., \& MACDonALD, J. (1976). Hearing lips and seeing voices. Nature, 264, 746-748.

Middleweerd, M. J., \& Plomp, R. (1987). The effect of speechreading on the speech-reception threshold of sentences in noise. Journal of the Acoustical Society of America, 82, 2145-2147.

Pearson, D. E. (1981). Visual communication system for the deaf. IEEE Transactions on Communications, COM-29, 1986-1992.

Reisberg, D., McLean, J., \& Goldfield, A. (1987). Easy to hear but hard to understand: A lip-reading advantage with intact auditory stimuli. In B. Dodd \& R. Campbell (Eds.), Hearing by eye: The psychology of lip-reading (pp. 97-113). London: Erlbaum.

RHoDEs, G. (1988). Looking at faces: First-order and second-order features as determinants of facial appearance. Perception, 17, 43-63.

Rosenblum, L. D., Johnson, J., \& Saldaña, H. M. (1996). Visual kinematic information for embellishing speech in noise. Journal of Speech \& Hearing Research, 39, 1159-1170.

Rosenblum, L. D., \& Saldaña, H. M. (1992). Discrimination tests of visually influenced syllables. Perception \& Psychophysics, 4, 461473.

Rosenblum, L. D., \& Saldaña, H. M. (1996). An audiovisual test of kinematic primitives for visual speech perception. Journal of Experimental Psychology: Human Perception \& Performance, 22, 318-331.

Rosenblum, L. D., Yakel, D. A., \& Green, K. P. (2000). Face and mouth inversion effects on visual and audiovisual speech perception. Journal of Experimental Psychology: Human Perception \& Performance, 26, 806-819.
SeArcy, J. H., \& Bartlett, J. C. (1996). Inversion and processing of component and spatial-relational information in faces. Journal of Experimental Psychology: Human Perception \& Performance, 22, 904915.

Sergent, J. (1984). An investigation into component and configurational processes underlying face perception. British Journal of Psychology, 75, 221-242.

Sumby, W.H., \& Pollack, I. (1954). Visual contribution to speech intelligibility in noise. Journal of the Acoustical Society of America, 26, 212-215

TAnaka, J. W., \& Farah, M. (1993). Parts and wholes in face recognition. Quarterly Journal of Experimental Psychology, 46A, 225-245.

VALENTINE, T. (1988). Upside-down faces: A review of the effect of inversion upon face recognition. British Journal of Psychology, 79, 471491.

VITKOVITCH, M., \& BARBER, P. (1996). Visible speech as a function of image quality: Effects of display parameters on lipreading ability. Applied Cognitive Psychology, 10, 121-140.

Walden, B. E., Prosek, R. A., Montgomery, A. A., Scherr, C. K., \& JoNES, C. J. (1977). Effects of training on the visual recognition of consonants. Journal of Speech \& Hearing Research, 20, 130-145.

Wallbott, H. G. (1992). Effects of distortion of spatial and temporal resolution of video stimuli on emotional attributions. Journal of Nonverbal Behavior, 16, 5-20.

(Manuscript received September 13, 2000; revision accepted for publication November 15, 2001.) 